\title{
Degenerate Curve Bifurcations in 3D Linear Symmetric Tensor Fields
}

\author{
Yue Zhang, Hongyu Nie, and Eugene Zhang
}

\begin{abstract}
D symmetric tensor fields have a wide range of applications in medicine, science, and engineering. The topology of tensor fields can provide key insight into their structures. In this paper we study the number of possible topological bifurcations in $3 \mathrm{D}$ linear tensor fields. Using the linearity/planarity classification and wedge/trisector classification, we explore four types of bifurcations that can change the number and connectivity in the degenerate curves as well as the number and location of transition points on these degenerate curves. This leads to four types of bifurcations among nine scenarios of 3D linear tensor fields.
\end{abstract}

\section{Introduction}

Tensor field visualization is an important topic in visualization, with many applications in medical imaging, solid and fluid mechanics, material science, earthquake engineering, and computer graphics.

Recent advances on tensor field visualization focus in topology-driven analysis and visualization of 3D symmetric tensor fields. Degenerate curves are one of the most fundamental topological features in a tensor field, and much research has focused on the understanding and efficient extraction of degenerate curves from piecewise linear tensor fields defined on a tetrahedral mesh [6-8].

\footnotetext{
Y. Zhang $(\bowtie)$

School of Electrical Engineering and Computer Science 3117 Kelley Engineering Center, Oregon State University, Corvallis, OR 97331, USA

e-mail: zhangyue@eecs.oregonstate.edu

H. Nie

School of Electrical Engineering and Computer Science 1148 Kelley Engineering Center, Oregon State University, Corvallis, OR 97331, USA

e-mail: nieh@oregonstate.edu

E. Zhang

School of Electrical Engineering and Computer Science 2111 Kelley Engineering Center, Oregon State University, Corvallis, OR 97331, USA

e-mail: zhange@eecs.oregonstate.edu 
In the book chapter, we focus on a problem that has received relatively little attention: bifurcations in tensor field topology. To make our investigation effective with potential application to real datasets, we focus on $3 \mathrm{D}$ linear tensor fields. We explore all the theoretically possible bifurcations. Moreover, we have conducted experiment to verify whether these bifurcations can occur.

The rest of the paper is structured as follows. Section 2 reviews past research in topology-driven analysis of symmetric tensor fields. In Sect. 3 we review relevant mathematical background and results on tensor fields. In Sect. 4 we report the findings of our exploration before concluding in Sect. 5.

\section{Previous Work}

Much research exists on 2D and 3D symmetric tensor fields, and we refer the readers to the survey by Kratz et al. [4] and Zhang et al. [11] for a more comprehensive review. In this book chapter we only refer to the research that is most relevant.

Delmarcelle and Hesselink [1] introduce the notion of degenerate points for 2D symmetric tensors, where eigenvector directions are not well-defined. Zhang et al. [12] explore the physical meanings of degenerate points in the stress tensor and strain tensor from continuum mechanics.

Hesselink et al. later extend this work to 3D symmetric tensor fields [3] and study the degeneracies in such fields. Zheng and Pang [16] point out that triple degeneracies are structurally unstable features. That is, an arbitrarily small perturbation to the field will remove such degenerate points. Zheng and Pang further show that double degeneracies, i.e., only two equal eigenvalues, form lines in the domain. In this work and subsequent research [18], they provide a number of degenerate curve extraction methods based on the analysis of the discriminant function of the tensor field. Furthermore, Zheng et al. [17] point out that near degenerate curves the tensor field exhibits 2D degenerate patterns and define separating surfaces which are extensions of separatrices from 2D symmetric tensor field topology. Tricoche et al. [9] convert the problem of extracting degenerate curves in a 3D tensor field to that of finding the ridge and valley lines of an invariant of the tensor field, thus leading to a more robust extraction algorithm. More recently, Palacios et al. [6] extract degenerate curves using an algorithm for algebraic surface extraction method called A-patches. Palacios et al. [5] introduce a number of topological editing operations with which a 3D tensor field can be edited for graphics applications.

Zhang et al. [13] describe a number of important properties of 3D linear tensor fields. They [15] show that in a 3D linear tensor field, there are at least two and at most four degenerate curves. Roy et al. [8] develop a parameterization with which all degenerate points in a 3D piecewise linear tensor field can be extracted efficiently and at any given accuracy. Zhang et al. [14] show that there are at most eight transition points in a 3D linear tensor field. 


\section{Background on Tensors and Tensor Fields}

In this section we review the most relevant background on $2 \mathrm{D}$ and $3 \mathrm{D}$ symmetric tensors and tensor fields [14].

\subsection{Tensors}

A $K$-dimensional (symmetric) tensor $\mathbf{T}$ has $K$ real-valued eigenvalues: $\lambda_{1} \geq \lambda_{2} \geq$ $\ldots \geq \lambda_{K}$. The largest and smallest eigenvalues are referred to as the major eigenvalue and minor eigenvalue, respectively. When $K=3$, the middle eigenvalue is referred to as the medium eigenvalue. An eigenvector belonging to the major eigenvalue is referred to as a major eigenvector. Medium and minor eigenvectors can be defined similarly. Eigenvectors belonging to different eigenvalues are mutually perpendicular.

The trace of a tensor $\mathbf{T}=\left(\mathbf{T}_{i j}\right)$ is $\operatorname{trace}(\mathbf{T})=\sum_{i=1}^{K} \lambda_{i} . \mathbf{T}$ can be uniquely decomposed as $\mathbf{D}+\mathbf{A}$ where $\mathbf{D}=\frac{\operatorname{trace}(\mathbf{T})}{K} \mathbb{I}(\mathbb{I}$ is the $K$-dimensional identity matrix) and $\mathbf{A}=\mathbf{T}-\mathbf{D}$. The deviator $\mathbf{A}$ is a traceless tensor, i.e., $\operatorname{trace}(\mathbf{A})=0$. Note that $\mathbf{T}$ and A have the same set of eigenvectors. Consequently, the anisotropy in a tensor field can be defined in terms of its deviator tensor field. Another nice property of the set of traceless tensors is that it is closed under matrix addition and scalar multiplication, making it a linear subspace of the set of tensors.

The magnitude of a tensor $\mathbf{T}$ is $\|\mathbf{T}\|=\sqrt{\sum_{1 \leq i, j \leq K} T_{i j}^{2}}=\sqrt{\sum_{i}^{K} \lambda_{i}^{2}}$, while the determinant is $|\mathbf{T}|=\prod_{i=1}^{K} \lambda_{i}$.

A tensor is degenerate when there are repeating eigenvalues. In this case, there exists at least one eigenvalue whose corresponding eigenvectors form a higherdimensional space than a line. When $K=2$ a degenerate tensor must be a multiple of the identity matrix.

\subsection{Tensor Field Topology}

We now review tensor fields, which are tensor-valued functions over some domain $\Omega \subset \mathbb{R}^{K}$. A tensor field can be thought of as $K$ eigenvector fields, corresponding to the $K$ eigenvalues. A hyperstreamline with respect to an eigenvector field $e_{i}(p)$ is a $3 D$ curve that is tangent to $e_{i}$ everywhere along its path. Two hyperstreamlines belonging to two different eigenvalues can only intersect at the right angle, since eigenvectors belonging to different eigenvalues must be mutually perpendicular.

Hyperstreamlines are usually curves. However, they can occasionally consist of only one point, where there is more than one choice of lines that correspond to the eigenvector field. This is precisely where the tensor field is degenerate. A point 

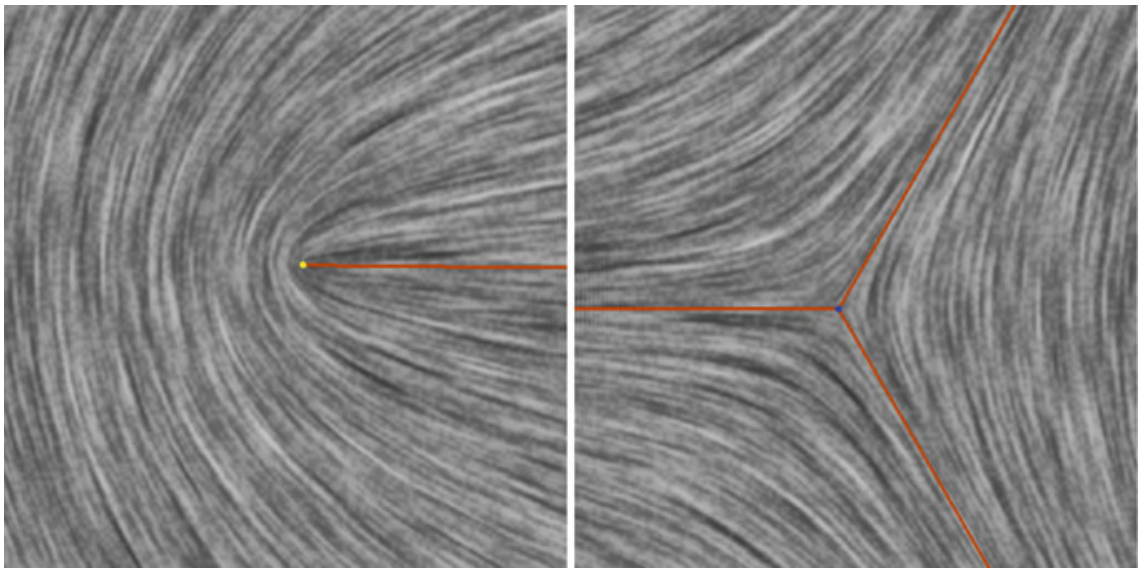

Fig. 1 A wedge (left) and a trisector (right)

$p_{0} \in \Omega$ is a degenerate point if $\mathbf{T}\left(p_{0}\right)$ is degenerate. One important topological feature of a tensor field consists of its degenerate points.

In $2 D$, the set of degenerate points of a tensor field consists of isolated points under numerically stable configurations, when the topology does not change given sufficiently small perturbation in the tensor field. An isolated degenerate point can be measured by its tensor index [10], defined in terms of the winding number of one of the eigenvector fields on a loop surrounding the degenerate point. The most fundamental types of degenerate points are wedges and trisectors, with a tensor index of $\frac{1}{2}$ and $-\frac{1}{2}$, respectively. Let $L \mathbf{T}_{p_{0}}(p)$ be the local linearization of $\mathbf{T}(p)$ at a degenerate point $p_{0}=\left(\begin{array}{l}x_{0} \\ y_{0}\end{array}\right)$, i.e.,

$$
L \mathbf{T}_{p_{0}}(p)=\left(\begin{array}{ll}
a_{11}\left(x-x_{0}\right)+b_{11}\left(y-y_{0}\right) & a_{12}\left(x-x_{0}\right)+b_{12}\left(y-y_{0}\right) \\
a_{12}\left(x-x_{0}\right)+b_{12}\left(y-y_{0}\right) & a_{22}\left(x-x_{0}\right)+b_{22}\left(y-y_{0}\right)
\end{array}\right)
$$

Then $\delta=\left|\left(\begin{array}{ll}\frac{a_{11}-a_{22}}{2} & a_{12} \\ \frac{b_{11}-b_{22}}{2} & b_{12}\end{array}\right)\right|$ is invariant under the change of basis [2]. Moreover, $p_{0}$ is a wedge when $\delta>0$ and a trisector when $\delta<0$. When $\delta=0, p_{0}$ is a higherorder degenerate point. A major separatrix is a hyperstreamline emanating from a degenerate point following the major eigenvector field. A minor separatrix is defined similarly.

The total tensor index of a continuous tensor field over a two-dimensional manifold is equal to the Euler characteristic of the underlying manifold. Consequently, it is not possible to remove one degenerate point. Instead, a pair of degenerate points with opposing tensor indexes (a wedge and trisector pair) must be removed simultaneously [10]. Figure 1 shows a wedge pattern (left) and a trisector pattern (right), respectively. 
In 3D, a degenerate point can have either two or three eigenvalues being the same, the latter of which is structurally unstable. Structurally stable degenerate points have two eigenvalues being the same. These eigenvalues are the repeating eigenvalues, while the third eigenvalue is the dominant eigenvalue. When the dominant eigenvalue is the major eigenvalue, the degenerate point is a linear degenerate point $(\mathrm{L})$. On the other hand, when the dominant eigenvalue is the the minor eigenvalue, the degenerate point is a planar degenerate point $(\mathrm{P})$. We refer to this linear/planar classification of a degenerate point as its L/P classification. Along a degenerate curve, its L/P classification does not change.

A degenerate point can also be classified in a different way, by projecting the tensor field onto the plane passing through the degenerate point and perpendicular to its dominant eigenvector. The degenerate point is also a degenerate point in the $2 \mathrm{D}$ projected tensor field. Consequently, the original 3D degenerate point is classified as either a wedge $(\mathrm{W})$ or a trisector $(\mathrm{T})$ based on the W/T type of the 2D degenerate point. Note that the projection of the 3D tensor field onto other planes not perpendicular to the dominant eigenvector does not necessarily have the same W/T classification. Along a degenerate curve, the W/T type can change, separated by degenerate points that are neither wedges nor trisectors. Such points are transition points.

Figure 2 illustrates these concepts with one example tensor field. The combination of the L/P and W/T classifications of a degenerate point leads to four combinations: LW (green), LT (blue), PW (yellow), and PT (red). Note that along a degenerate curve, the L/P classification is constant while the $\mathrm{W} / \mathrm{T}$ classification can change. Therefore, a degenerate curve can consist of either green and blue segments, or yellow and red segments, but not other combinations of colors. Linear transition points appear between green and blue segments, while planar transition points separate yellow and red segments.

The projected tensor fields onto the plane of repeating eigenvalues are shown along the degenerate curve. Notice that at a $\mathrm{W}$ type point, the projected pattern shows a wedge, while at a $\mathrm{T}$ type point, the projected pattern shows a trisector. At a transition point, the projected pattern shows neither a wedge pattern nor a trisector pattern.

\subsection{D Linear Tensor Fields}

A 3D linear tensor field is a 3D symmetric tensor field whose tensor entries are linear functions of the $\mathrm{XYZ}$ coordinates of the points in the domain. It has the following form:

$$
T(x, y, z)=T_{0}+x T_{x}+y T_{y}+z T_{z}
$$

where $T_{0}, T_{x}, T_{y}$, and $T_{z}$ are 3D symmetric tensors. A 3D linear tensor field has all the aforementioned properties of a general tensor field. However, there are some important properties specific to 3D linear tensor fields. 


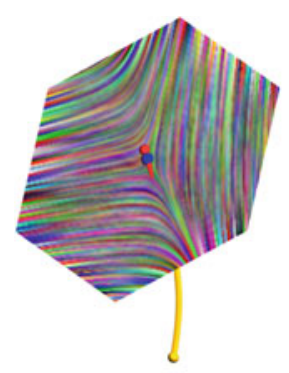

(a)

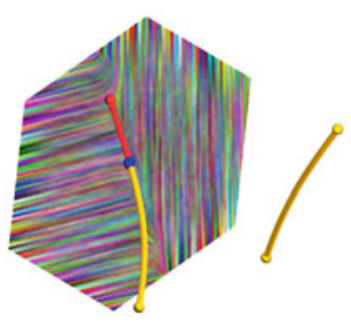

(b)

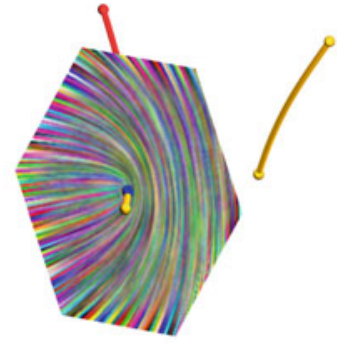

$(c)$

Fig. 2 Along a degenerate curve, the projection of the tensor field onto the repeating planes can exhibit 2D degenerate patterns such as a trisector (a) or a wedge (c). Between segments of wedges (yellow) and trisectors (red), transition points can appear (b)

First, there are either two or four degenerate curves in a 3D linear tensor field [15]. Half of these curves are L, and the other half are P.

Second, there are either zero, two, four, six, or eight transition points in a 3D linear tensor field [14].

As pointed out [8], the set of degenerate points in a 3D linear tensor field (including their asymptotic limits at infinity) is homeomorphic to a topological circle (a loop). Figure 3 illustrates this bijective map. The loop is situated on the unit sphere where the location of a degenerate point on the loop corresponds to its unit dominant eigenvector. Since eigenvectors have a sign ambiguity, there are two such loops. Every degenerate point corresponds to exactly one pair of antipodal points on the sphere. While L/P cannot change along a degenerate curve, such a switch can occur at infinity, which two degenerate curves approach in opposite directions. The number of such switch points, called $\infty$ points, is the same as the number degenerate curves in the field. Note that $\infty$ points are neither L nor P. On the other hand, they can be either W or T. This leads to nine scenarios [14]: (1) two WW curves, (2) two WT curves, (3) two TT curves, (4) four WW curves, (5) two WW curves and two WT curves, (6) one WW curve, two WT curves, and one TT curve, (7) four WT curves, (8) two WT curves and two TT curves, and (9) four TT curves.

Along a $W W$ or $T T$ curve, there must be an even number of transition points, while on a $W T$ curve there must exist an odd number of transition points.

The color of the sphere represents the sign of the discriminant of the projected tensor field onto the plane whose normal is the displacement vector of the point on the sphere from the center of the sphere. The color is cyan if the discriminant is positive (wedge type) and magenta if the discriminant is negative (trisector type). Note the W/T classification of a degenerate point must match the sign of the discriminant on the sphere. That is, green and yellow segments must appear in the cyan region, while blue and red segments must appear in the magenta region. A transition point must appear on the boundary between cyan and magenta regions. 

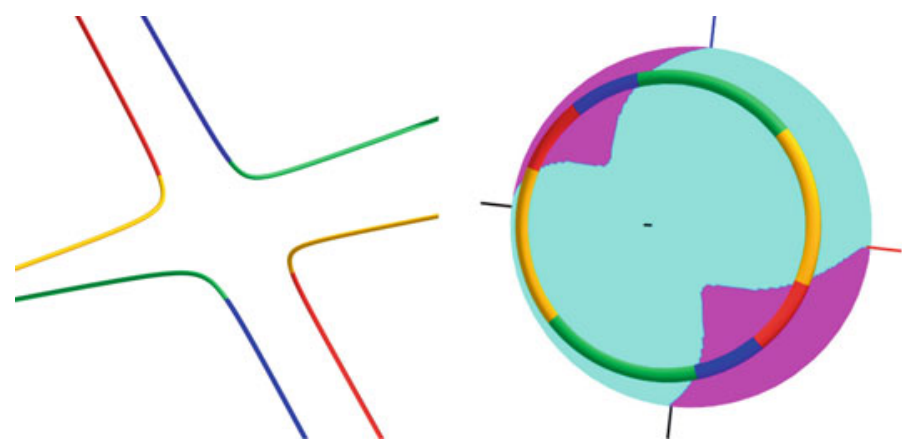

Fig. 3 This figure shows the parameterization of all degenerate points in a 3D linear tensor field by a topological circle. In the left are the degenerate curves of a 3D linear tensor field, and in the right is the topological circle superimposed on the unit sphere. Between the yellow and green segments are $\infty$ points. In addition, transition points occur either between yellow and red segments, or between green and blue segments. Moreover, we color a point in the sphere (right: representing a unit vector) cyan if the tensor projected onto the plane perpendicular to this vector has a wedge degenerate point. On the other hand, the point is colored magenta if the projected tensor field has trisector degenerate point. Notice that the transition points on the degenerate curves (between blue and green segments and between yellow and red segments) are precisely on the boundary between the cyan and magenta regions

\section{Bifurcations}

We now describe our findings of bifurcations in 3D linear tensor fields.

First, there can be either two or four degenerate curves. This means that some bifurcations can change the number of degenerate curves in the field. We refer to those bifurcations which reduce the number of degenerate curves from four to two as degenerate curve removal and those increase the number from two to four as degenerate curve generation.

Second, even when the number of degenerate curves does not change, the way the $\infty$ points are connected can change. We refer to this as degenerate curve reconnection, which is consistent with the topological editing operation of the same name from [5]. Note that two degenerate curves of opposite L/P types cannot be reconnected as this would generate a degenerate curve with changing L/P type. Consequently, when there are only two degenerate curves in the field, they cannot be connected. Degenerate curve reconnection can only occur for four degenerate curves.

Third, the number of transition points in the tensor field can change. Since this number must be even, we refer to such bifurcations as either transition point pair cancellation if the number is decreased by two or transition point pair generation if the number is increased by two.

Finally, even when the number of transition point does not change in a field, it is possible that some transition point has been moved from one degenerate curve to another. We refer to such a bifurcation as a transition point relocation.

Below we consider these bifurcations in the context of the nine scenarios described earlier. 


\subsection{Degenerate Curve Removal and Generation}

Note that a degenerate curve generation bifurcation must be the inverse of a degenerate curve removal bifurcation, and vice versa. Consequently, we consider them together in this section.

Figure 4 lists all theoretically possible degenerate curve generation bifurcations and their inverse bifurcations. There is a total of nine scenarios, each of which is represented as an ellipse (topological disk) with the $\infty$ points marked along with their W/T types. A degenerate curve removal bifurcation must take a scenario with four degenerate curves (four $\infty$ points on the ellipse) to one with two degenerate curves (two $\infty$ points). Conversely, a degenerate curve generation bifurcation must take a scenario with two degenerate curves to one with four degenerate curves. In addition, when two degenerate curves are removed, two adjacent $\infty$ points are removed and two $\infty$ points remain. Each of the $\infty$ point is written as either $\mathrm{W}$ or $\mathrm{T}$ (called a symbol on the ellipse). This means that not any four-symbolled ellipse can connect with any two-symbolled ellipse. For example, while the box with four $W$ 's can be mapped to either one with two $W$ 's or one with one $W$ and one $T$, it can not be connected with one with two $T$ 's. Similarly, the box with one $W$ and three $T$ 's cannot be mapped to one with two $W$ 's. Furthermore, when two symbols are removed, they need to be adjacent symbols. Consequently, it is not possible to connect the ellipse with two $W$ 's and two $T$ 's that are interleaved to one with two $W$ 's or the one with two $T$ 's.

These constraints give rise to a total of ten theoretically possible bifurcations, each of which is given as an edge in Fig. 4. The following figures provide examples of degenerate curve removal bifurcations.

In Fig. 5 (left), there are initially four TT curves, two of which are linear (blue) and two planar (red). After the bifurcation (right), the two linear degenerate curves become connected by a linear wedge segment (green), which replaces the lost planar

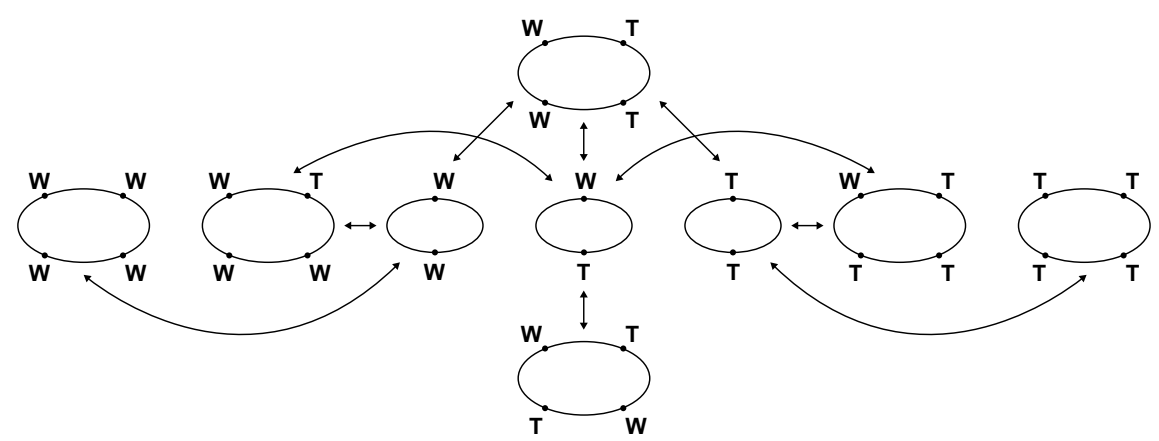

Fig. 4 This diagram shows all the theoretically possible degenerate curve removal and generation bifurcations in a 3D linear tensor field 


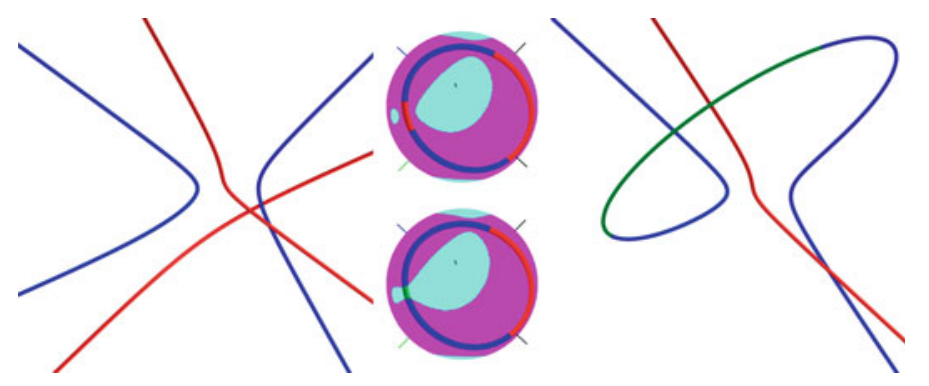

Fig. 5 This figure shows a degenerate curve removal bifurcation from the case of four TT curves (left) to two TT curves (right). The top-middle shows the non-repeating eigenvector manifold for the left, while the bottom-middle shows the non-repeating eigenvector manifold for the right. The inverse bifurcation, a degenerate curve generation exists, which swaps the before and after scenarios. Note that in these bifurcations the number of segments do not change after the bifurcation

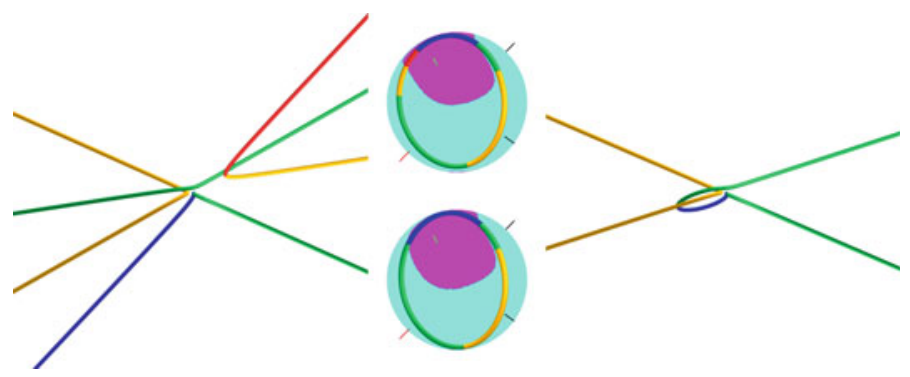

Fig. 6 Another degenerate curve removal bifurcation takes the case of two WW curves and two WT curves (left) to two WW curves (right). Note that the number of segments is decreased by two after the bifurcation, i.e. two transition points are lost as a result of the bifurcation

TT curve (red). Furthermore, two transition points appear on the newly connected linear degenerate curve.

The middle column compares the two fields using their parameterization: (uppermiddle) corresponding to the field in the left, and (lower-middle) corresponding to the tensor field in the right. Through the bifurcation, two infinity points are replaced by two transition points, resulting in the switch of a red segment (middle-top) to a green segment (middle-bottom), which leads to the loss of two degenerate curves and the creation of two transition points.

Figure 6 provide an additional example of such types of bifurcations. Note that in this example, the loss of two degenerate curves also results in the loss of two transition points, i.e. the loss of two colored segments. This is a different way of achieving degenerate curve removal than the example bifurcation in Fig. 5, in which the number of segments does not change. 


\subsection{Degenerate Curve Reconnection}

Degenerate curve reconnection bifurcations do not change the number of degenerate curves. Instead, they change how the $\infty$ points are connected. As mentioned earlier, two degenerate curves can be connected only if they have the same L/P type. When there are only two degenerate curves, one is $\mathrm{L}$ and the other $\mathrm{P}$, which cannot be connected after reconnection. Consequently, degenerate curve reconnection bifurcations only occur for the case of four degenerate curves.

There is a total six scenarios with four degenerate curves, as shown in Fig. 7. Since degenerate curve reconnection bifurcations do not change the number and type of $\infty$ points, there is at least one theoretically possible bifurcation that connects a scenario to itself. Furthermore, the two scenarios with two $W$ 's and two $T$ 's are connected through reconnection bifurcations.

However, it is worth noting that when reconnection occurs, the $\infty$ points on the two degenerate curves to be reconnected cannot be arbitrarily connected after bifurcation. For an example, consider the case of two $W$ 's and two $T$ 's that appear on the ellipse in an alternating fashion (the lower-middle ellipse) in Fig. 7. Suppose the top and bottom segments are to be reconnected while the left and right segment remain. Then one cannot connect the upper-left $W \infty$ point with the lower-left $T$ $\infty$ point, as it would cause the ellipse to be broken into two topological disks, a structurally unstable case. This means that it is not possible to have a reconnection bifurcation that takes this scenario to itself. Instead, the only way to reconnect is to connect the upper-left $W \infty$ point with the lower-right $W \infty$ point and connect the upper-right $T \infty$ point with the lower-left $T \infty$ point, leading to the scenario of two adjacent $W$ and two adjacent $T \infty$ points on the ellipse (the upper-middle ellipse).

In fact, the above argument is true in general. That is, when reconnecting, the upper-left $\infty$ point must be connected to the lower-right $\infty$ point, and the upperright $\infty$ point must be connected to the lower-left $\infty$ point. This analysis shows that there are five degenerate curve reconnection bifurcations that takes one scenario to itself, and there are two more that occur between two different scenarios (the upper-middle ellipse and the lower-middle ellipse).

Figure 8 shows the reconnection in the scenario of four WW curves, in which two linear degenerate curves (green) are reconnected, resulting in two linear degenerate curves. The two planar degenerate curves (yellows) are not reconnected. This bifurcation does not increase or decrease the number of transition points.

Figure 9 presents another degenerate curve reconnection bifurcation in which two planar WT degenerate curves are reconnected into one planar WW degenerate curve and one planar TT degenerate curve. As a result of this bifurcation, two transition points are removed, which leads to a decrease in the total number of transition points in the field. Notice the order of colored segments (middle column of Fig. 9) is changed due to the reconnection. 

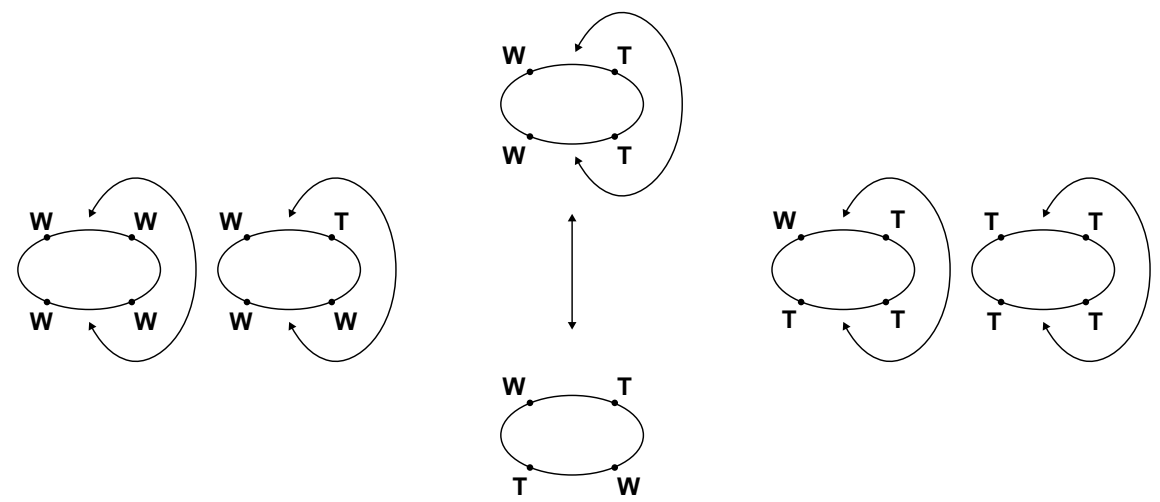

Fig. 7 This diagram shows all the possible degenerate curve reconnection bifurcations in a 3D linear tensor field

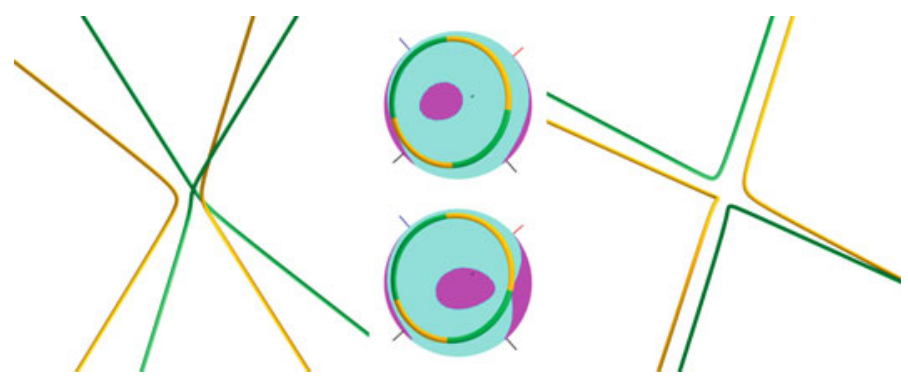

Fig. 8 This figure shows a degenerate curve reconnection bifurcation from the case of four WW curves (left) to four WW curves (right). The number of transition points does not change after the bifurcation

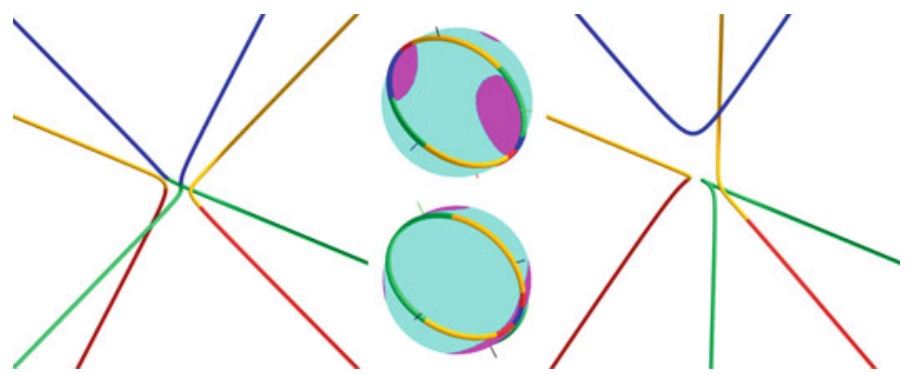

Fig. 9 Another degenerate curve reconnection bifurcation takes the case of one WW curve, 2WT curves, and one TT curve (left) to four WT curves (right). In this case, the number of degenerate curve bifurcation is decreased by two 


\subsection{Transition Point Pair Cancellation and Generation}

The total number of transition points in a 3D linear tensor field is an even number between zero and eight. That means that any fundamental bifurcation that changes the number of total transition points must either increase it by two or decrease it by two. The former is the degenerate curve pair generation bifurcation, and the latter degenerate curve pair cancellation bifurcation. They are inverse bifurcations and thus discussed together in this section.

Two examples of transition point pair cancellation bifurcations are shown in Figs. 11 and 12, for the cases of two degenerate curves and four degenerate curves, respectively. The transition point pair cancellation and generation bifurcations do not change the scenario. Consequently, for each of the nine scenarios, there is a bifurcation that takes this scenario to itself, as shown in Fig. 10. There is a total of nine transition point pair cancellation bifurcations and nine transition point pair generation bifurcations.

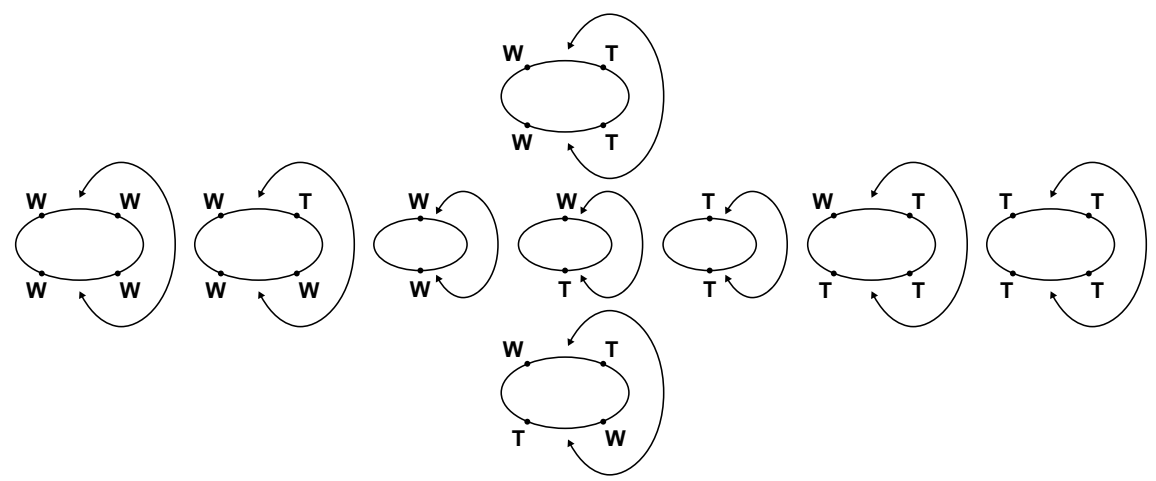

Fig. 10 This diagram shows all the possible transition pair cancellation and generation bifurcations in a $3 \mathrm{D}$ linear tensor field for one degenerate curve

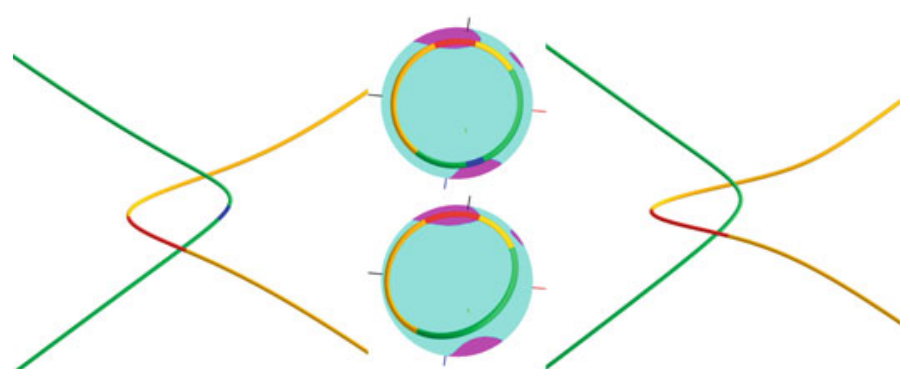

Fig. 11 A transition point pair cancellation bifurcation that removes two transition points and one blue segment from one of the two degenerate curves (left), resulting in the same number of degenerate curves with two fewer transitions points (right) 


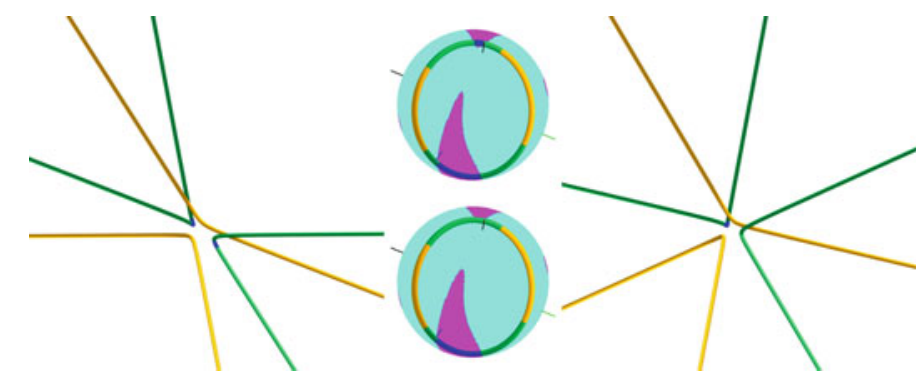

Fig. 12 A transition point pair cancellation operation (from to right) occurs in the case of four degenerate curves

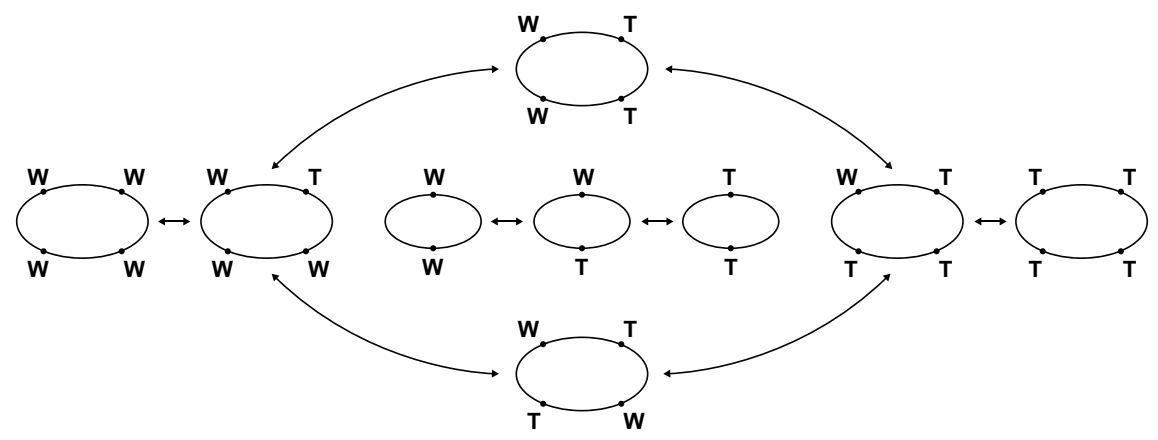

Fig. 13 This diagram shows all the possible transition point relocation bifurcations in a 3D linear tensor field. Note that these bifurcations have the effect of switching one $\mathrm{W}$ symbol to one $\mathrm{T}$ symbol, or vice versa. Consequently, there are only eight possible such bifurcations, all observed in our experiment

\subsection{Transition Point Relocation}

Transition points, along with $\infty$ points, divide the degenerate curves into segments of pure $\mathrm{L} / \mathrm{P}$ and $\mathrm{W} / \mathrm{T}$ types. While the total number of transition points in a 3D linear tensor field is eight, its distribution is not uniform among the degenerate curves.

Transition point relocation refers to moving one transition point from one degenerate curve to another degenerate curve of the opposite L/P type. From the viewpoint of the parameterization, this is the swap of the positions of the transition point with one adjacent infinity point on the ellipse in the counterclockwise order. This results in one fewer transition point on the original degenerate curve and one more transition point on the new degenerate curve but keeps the total number of transition points constant. Moreover, the W/T type of the segment also changes. Consequently, the segment between the transition point and infinity point will change its L/P type but maintains its W/T type, thus will change colors between red and green, or between yellow and blue. Figures 14 and 15 provide two examples of this type of bifurcations that involve respectively two degenerate curves and four degenerate curves. 


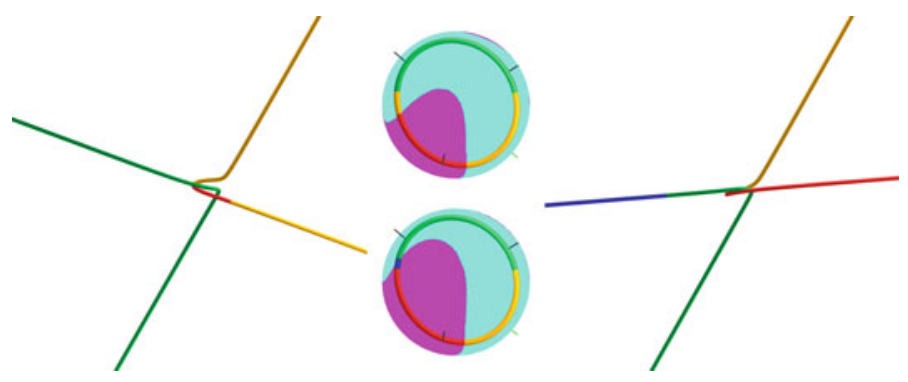

Fig. 14 This figure shows a transition point relocation bifurcation from the case of two WW curves (left) to two WT curves (right)
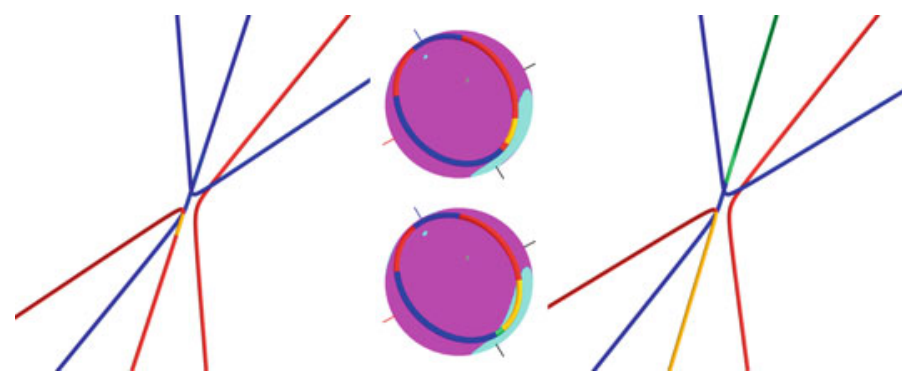

Fig. 15 Another transition point relation bifurcation takes the case of four TT curves (left) to two TT and two WT curves (right)

Figure 13 shows the possible transition point relation bifurcations. Note that these bifurcations result in a switch of a $\mathrm{W}$ infinity point to a $\mathrm{T}$ type of infinity point, or vice versa. Note that each edge in this diagram corresponds to two transition point relocation bifurcations. As there are eight edges in the diagram, there is a total of 16 bifurcations, all of which have been observed during experiment.

\section{Conclusion}

Degenerate curves and transition points play an important role in describing the behavior of a $3 \mathrm{D}$ tensor field. In this book chapter, we study possible bifurcations for any 3D linear tensor field.

This is done based on the realization that there is a total of nine scenarios and four types bifurcations. Theoretically, we have identified ten degenerate curve removal bifurcations, ten degenerate curve generation bifurcations, seven degenerate curve reconnection bifurcation, nine transition point pair cancellation bifurcations, nine transition point pair generation bifurcations, and 16 transition point relation bifurca- 
tions. This leads to a total of 61 topological bifurcations for 3D linear tensor fields, all of which have been observed.

There are a number of future research directions.

First, the number of transition points can further divide each scenario into subscenarios. For example, for the scenario of two $W W$ curves, there can be zero, two, four, six, or eight transition points. Furthermore, transition points are not evenly distributed on degenerate curves. This leads to multiple cases per scenario. Enumerating of all bifurcations from any sub-scenario to any other sub-scenario can lead to deeper insight about the topology of 3D symmetric tensor fields.

Second, the bifurcations among 3D tensor fields can be used to generate a multiscale framework for the topological analysis of these objects, such as degenerate curve clustering and removal. Such a framework has the potential of enabling users to inspect the topology of their tensor field data at various scales. We plan to investigate this possibility in our future research.

Third, simulation data sets usually involve piecewise linear tensor fields, which can have potentially more types of bifurcations than linear tensor fields that we investigate in this paper. This is a future direction that can lead to more practical applications for tensor field topology.

Fourth, neutral surfaces are the other important constituent of 3D tensor field topology. Enumerating the scenarios of neutral surfaces and bifurcations of these different scenarios can provide insight into the structure of a tensor field. We will explore this direction.

Acknowledgments We wish to thank our anonymous reviewers for their valuable feedback and suggestions. This research is partially supported by NSF awards (\# 1566236) and (\#1619383).

\section{References}

1. Delmarcelle, T., Hesselink, L.: Visualizing second-order tensor fields with hyperstream lines. IEEE Comput. Graph. Appl. 13(4), 25-33 (1993)

2. Delmarcelle, T., Hesselink, L.: The topology of symmetric, second-order tensor fields. In: Proceedings IEEE Visualization 1994 (1994)

3. Hesselink, L., Levy, Y., Lavin, Y.: The topology of symmetric, second-order 3D tensor fields. IEEE Trans. Visual. Comput. Graph. 3(1), 1-11 (1997)

4. Kratz, A., Auer, C., Stommel, M., Hotz, I.: Visualization and analysis of second-order tensors: Moving beyond the symmetric positive-definite case. Comput. Graph. Forum 32(1), 49-74 (2013). http://dblp.uni-trier.de/db/journals/cgf/cgf32.html\#KratzASH13

5. Palacios, J., Roy, L., Kumar, P., Hsu, C.Y., Chen, W., Ma, C., Wei, L.Y., Zhang, E.: Tensor field design in volumes. ACM Trans. Graph. 36(6), 188:1-188:15 (2017). https://doi.org/10.1145/ 3130800.3130844

6. Palacios, J., Yeh, H., Wang, W., Zhang, Y., Laramee, R.S., Sharma, R., Schultz, T., Zhang, E.: Feature surfaces in symmetric tensor fields based on eigenvalue manifold. IEEE Trans. Visual. Comput. Graph. 22(3), 1248-1260 (2016). https://doi.org/10.1109/TVCG.2015.2484343

7. Raith, F., Blecha, C., Nagel, T., Parisio, F., Kolditz, O., Günther, F., Stommel, M., Scheuermann, G.: Tensor field visualization using fiber surfaces of invariant space. IEEE Trans. Visual. Comput. Graph. 25(1), 1122-1131 (2019). https://doi.org/10.1109/TVCG.2018.2864846 
8. Roy, L., Kumar, P., Zhang, Y., Zhang, E.: Robust and fast extraction of 3D symmetric tensor field topology. IEEE Trans. Visual. Comput. Graph. 25(1), 1102-1111 (2019), (IEEE VisWeek 2018)

9. Tricoche, X., Kindlmann, G., Westin, C.F.: Invariant crease lines for topological and structural analysis of tensor fields. IEEE Trans. Visual. Comput. Graph. 14(6), 1627-1634 (2008). https:// doi.org/10.1109/TVCG.2008.148

10. Zhang, E., Hays, J., Turk, G.: Interactive tensor field design and visualization on surfaces. IEEE Trans. Visual. Comput. Graph. 13(1), 94-107 (2007)

11. Zhang, S., Kindlmann, G., Laidlaw, D.H.: Diffusion tensor MRI visualization. In: Visualization Handbook. Academic Press (2004). http://www.cs.brown.edu/research/vis/docs/pdf/Zhang2004-DTM.pdf

12. Zhang, Y., Gao, X., Zhang, E.: Applying $2 d$ tensor field topology to solid mechanics simulations. In: Schultz, T., Özarslan, E., Hotz, I. (eds.) Modeling, Analysis, and Visualization of Anisotropy, pp. 29-41. Springer International Publishing, Cham (2017)

13. Zhang, Y., Palacios, J., Zhang, E.: Topology of 3d linear symmetric tensor fields. In: Hotz, I., Schultz, T. (eds.) Visualization and Processing of Higher Order Descriptors for Multi-Valued Data, pp. 73-91. Springer International Publishing, Cham (2015)

14. Zhang, Y., Roy, L., Sharma, R., Zhang, E.: Maximum number of transition points in $3 \mathrm{~d}$ linear tensor fields. In: Carr, H., Fujishiro, I., Sadlo, F., Takahashi, S. (eds.) Topological Methods in Data Analysis and Visualization V, pp. 237-250. Springer International Publishing (2020)

15. Zhang, Y., Tzeng, Y.J., Zhang, E.: Maximum number of degenerate curves in $3 \mathrm{~d}$ linear tensor fields. In: Carr, H., Garth, C., Weinkauf, T. (eds.) Topological Methods in Data Analysis and Visualization IV, pp. 221-234. Springer International Publishing, Cham (2017)

16. Zheng, X., Pang, A.: Topological lines in $3 \mathrm{~d}$ tensor fields. In: Proceedings IEEE Visualization 2004, VIS 2004, pp. 313-320. IEEE Computer Society, Washington, DC, USA (2004). https:// doi.org/10.1109/VISUAL.2004.105

17. Zheng, X., Parlett, B., Pang, A.: Topological structures of 3D tensor fields. Proc. IEEE Visual. 2005, 551-558 (2005)

18. Zheng, X., Parlett, B.N., Pang, A.: Topological lines in 3d tensor fields and discriminant hessian factorization. IEEE Trans. Visual. Comput. Graph. 11(4), 395-407 (2005)

Open Access This chapter is licensed under the terms of the Creative Commons Attribution 4.0 International License (http://creativecommons.org/licenses/by/4.0/), which permits use, sharing, adaptation, distribution and reproduction in any medium or format, as long as you give appropriate credit to the original author(s) and the source, provide a link to the Creative Commons licence and indicate if changes were made.

The images or other third party material in this chapter are included in the chapter's Creative Commons licence, unless indicated otherwise in a credit line to the material. If material is not included in the chapter's Creative Commons licence and your intended use is not permitted by statutory regulation or exceeds the permitted use, you will need to obtain permission directly from the copyright holder.

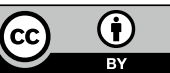

